\title{
Internacionalización de la educación superior
}

\author{
Blanca Ruth Orantes \\ Directora de Investigaciones Utec
}

\section{Resumen}

Las universidades se enfrentan a una nueva configuración del orden internacional en el ámbito de países e instituciones. Los modelos de producción innovadores impulsan a la ciencia y la tecnología. Las fronteras están desapareciendo y transformando los valores económicos, además de observar un crecimiento vertiginoso de la información y saberes. Todo lo anterior enfrenta a las universidades a una nueva exigencia, prepararse, crear una cultura para la internacionalización; y sus exigencias: idioma y tecnología de avanzada. Aún cuando se reconozca la importancia de la internacionalización y cooperación, se requiere de planes, acciones y presupuesto para su ejecución. No obstante, la cultura de internacionalización también se debe desarrollar por medio de un modelo que integre la voluntad, la capacidad y la identidad.

\section{Relaciones internacionales en las universidades}

as relaciones internacionales en las universidades son —instrumento importante, tanto para la búsqueda de cooperación internacional como para la internacionalización de la educación superior, y especialmente como apoyo a los proyectos de formación de capacidades en docencia e investigación. Esta necesidad se visualizó por un equipo de expertos y dio lugar al proyecto "Promoción de la internacionalización en Centroamérica", Inca, cuyos objetivos son potenciar la transferencia de conocimientos, técnicas de gestión y buenas prácticas para la gestión de las oficinas de relaciones internacionales.Lo que se busca es apoyar las relaciones internacionales de las universidades miembros. Está conformada por universidades públicas y privadas de cada país de América Central, incluyendo Panamá. Todas ellas bajo la coordinación de la Universidad de Alicante y el Instituto Tecnológico de Costa Rica. Se considera un proyecto exitoso, pues si bien algunas de las universidades participantes ya cuentan con oficinas de relaciones internacionales (ORI). Existen otras universidades que, realizan gestión internacional, esta se ejecuta de forma descentralizada. Para el último caso citado, Inmaculada Madera de la Universidad APES de Santo Domingo recomienda iniciar formando un comité institucional de Internacionalización permanente, luego realizar un diagnóstico, proceder a la conformación de una oficina de cooperación que inicie un proceso de formación, planes y

*Artículo basado en el encuentro de la Red Inca en El Salvador, América Central , sede UES - UTEC, 5, 6 y 7 de Julio de 2010. 
mecanismos de seguimiento hasta conformar la unidad vinculada con todas las instancias de la universidad. A partir de ello, se debe iniciar todo un proceso de Marketing (MKT).

Roberto Escarré de la Universidad de Alicante, España, considera que el MKT vende la imagen de la universidad. Pero que se debe desarrollar MKT de alta tecnología para poder competir en el mercado internacional, con una organización educativa orientada al cliente internacional; crear factores de atracción para ese cliente. Los nichos de mercados deben focalizarse y ofrecer planes o cursos atractivos. Por ejemplo un "curso de español para japoneses más un curso de fútbol" (caso España). Todo debe ser parte de las estrategias, de acuerdo con las condiciones de cada país o región. Al respecto, Gaspar Hernández, de Alicante, explica que dentro de las estrategias se debe informar sobre las actividades y proyectos internacionales, orientar el servicio educativo a estudiantes extranjeros, diseñar un programa para acogida de visitantes internacionales y evaluar la rentabilidad de los servicios. la Universidad de Alicante tiene 50 titulaciones y más de 30 mil estudiantes. Hay mucha movilidad e intercambio, se tiene un campus innovador. La oficina de relaciones internacionales realiza promoción, a escala internacional, de la oferta académica y de la conveniencia de estancia para estudiantes extranjeros, promoviendo a la vez productos o servicios atractivos. Se procura que los planes y el currículo sean de calidad. La oficina también coordina programas de movilidad de nacionales y extranjeros. Además, impulsa iniciativas en la búsqueda de acciones de cooperación para el desarrollo.

\section{Universidades de El Salvador que pertenecen a Inca}

Ada Ruth Nieto explicó el quehacer de las oficinas de relaciones internacionales de la Universidad de El Salvador (UES). Tienen como objetivo mejorar los procesos de gestión de cooperación internacional como medio para potenciar la do- cencia, la investigación y la proyección social. Actualmente la oficina cuenta con nueve empleados y catorce estudiantes como colaboradores. Explicó que se está trabajando por una mayor integración y colaboración por parte de algunas unidades de la universidad. Explicó que había una estructura por área geográfica, por ejemplo, Estados Unidos y Canadá, Asia, Europa, Latinoamérica, entre otros y alrededor de ellas se planifican las acciones.

La universidad Tecnológica de El Salvador (Utec), dio a conocer en el evento su quehacer internacional, como parte de sus actividades. Blanca Ruth Orantes expuso que se cuenta con líneas estratégicas centradas en gestión de la calidad, consolidación de vínculos, internacionalización de la enseñanza y búsqueda de cooperación internacional. Actualmente la Utec está fuertemente vinculada con la comunidad educativa y la sociedad salvadoreña, ya que se cuenta con más de 19 mil estudiantes de diversos sectores, pero que en su mayoría se concentran en la clase media baja, provenientes de sectores populosos de San Salvador y del interior de la República. En cuanto a la acreditación, existe un reconocimiento nacional e internacional, además de Inca, se participa en otras redes internacionales. La comunidad Utec tiene conciencia de la necesidad de cambios, en cuanto a la adaptación del nuevo orden internacional y la contribución al desarrollo nacional. Con el objeto de potenciar la internacionalización de la Utec, la gestión internacional ha generado convenios internacionales. Se cuenta con convenios firmados con universidades de prestigio extranjeras, que han dado resultados efectivos. Una declaración internacional para el Museo Universitario de Antropología, (MUA) programas de cartas de entendimiento y convenios que implican programas de movilidad e investigaciones conjuntas para docentes e investigadores, así como relación con organismos y embajadas. UTEC es sujeto de cooperación pues está inscrita en entes cooperantes, como ejemplo en Pador (Europa Programas de Cooperación Exterior) Permanente se 
aplica a proyectos conjuntos, vinculados a la Aecid, OEI, BID, Unesco, PNUD, Fundación Carolina, entre otros. Las acciones apuestan a la calidad en docencia, investigación y proyección social así como mejorar las capacidades de gestión internacional. Para el pasado año 2010, miembros de la comunidad Utec se encontraban en programas internacionales de doctorado, maestrías, postgrado y cursos. En los países de España, Costa Rica, Nicaragua, Guatemala y Estados Unidos, algunos de los centros Castilla-La Mancha, De Valencia, Granada, ITCR. Incae, Flacso, Salamanca, Fulbright, entre otros.

En el programa Visitantes académicos extranjeros distinguidos, se ha tenido en el 2009 y 2010 alrededor de quince visitantes, financiados por este programa, siendo el objetivo fortalecer la investigación, docencia y proyección social, así como observar las experiencias institucionales y profesionales de ellos hacia el sector docente e investigador. Se destacan la visita del Dr. José Padrón, de Venezuela, y el Dr. Paul Kim, de la Universidad de Stanford, California; el Dr. Claudio

\section{"Las relaciones internacionales}

\section{en las universidades}

son instrumento importante,

tanto para la búsqueda

de cooperación internacional

como para la internacionalización

de la educación superior,

y especialmente como apoyo

a los proyectos de investigación 77

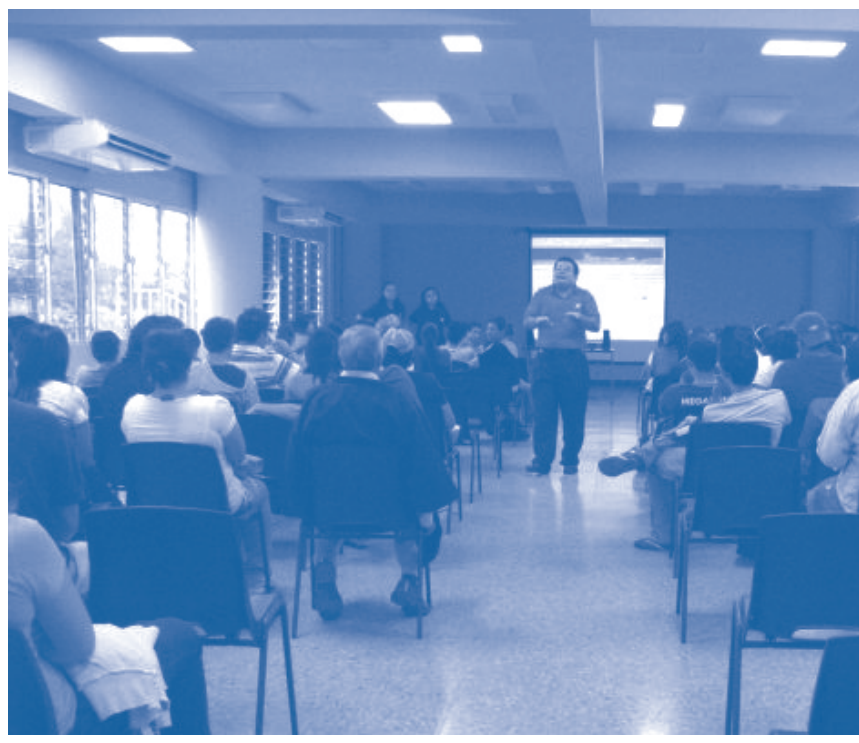

Rama, de Uruguay; el Dr. Ernesto Ayala, Dra. Clemencia Camacho, entre otros.

En cuanto a redes universitarias, la Utec pertenece a redes universitarias nacionales e internacionales: a) Nacionales Raices, Auprides b) Red Latinoamericana de Cooperación Universitaria, Red Urdimbre: Educación e Investigación, que reúne a trece universidades latinoamericanas, entre ellas la Universidad de Ibagué y el Politécnico Grancolombiano de Colombia, la Universidad de Guadalajara, la Universidad Nacional Abierta de Venezuela, entre otras. La Red Inca, es un proyecto cofinanciado por la Unión Europea en el marco del programa Alfa. Es la Promoción de la Internacionalización en América Central, conformada por doce universidades, de seis países, coordinadas por la Universidad de Alicante y el Instituto Tecnológico de Costa Rica. El Salvador, participa por medio de la Universidad de El Salvador y la Universidad Tecnológica de El Salvador. Es un proyecto que ofrece la oportunidad de potenciar la internacionalización y conformar una red de cooperación entre las universidades que contribuye a la región. Existen otras actividades que se reflejan en el proceso de internacionalización que la Utec ha iniciado, y que ya se observan buenos resultados: a) En investigación: formación de investigadores, consolidación de grupos de 
investigación, investigaciones conjuntas y publicaciones. B) Docencia: transferencia de experiencias, incremento e innovación del conocimiento, investigación formativa como eje transversal en el proceso enseñanza-aprendizaje. C) Gestión: buenas prácticas, capacidad de gestión, transferencia de know-how y consolidación de vínculos.

\section{Algunas experiencias de las oficinas de rela- ciones internacionales en universidades que pertenecen a la Red Inca}

Zizar Ayadi, de la Universidad de Alicante, explicó que la búsqueda de cooperación es la clave en el diseño de estrategias y búsqueda de socios. La negociación debe darse por expertos y bajo el principio de reciprocidad o ventajas comparativas de las instituciones. Además de considerar las variables gestión transnacional y la dimensión humana. Sobre ello se planifica, se mantiene un control y coordinación de las acciones internacionales. Los errores y defectos por evitar son: pensar solo en la financiación, no trabajar para resolver problemas o necesidades de contraparte, no asumir los proyectos, no trabajar en equipo, no contar con equipo preparado, no asumir riesgos, entre otros. Networking y búsqueda de socios. Es encontrar rápidamente a quién necesitas. Dar respuestas en tiempos mínimos, presentar ideas realistas, saber escuchar, no hacer juicios previos.

Odilia Mitre, de la Universidad Tecnológica de Panamá, señaló la importancia del papel que desempeñan el personal que integra las oficinas de relaciones internacionales, principalmente el del director de cooperación. Deben ser personas que resuelven problemas de la comunidad educativa Help Desk. Debe ser un servicio que orienta y gestiona para toda la comunidad, pero principalmente que integre el trabajo. La oficina de relaciones internacionales se encarga de ello, orienta y gestiona la movilidad internacional a estudiantes, docentes y autoridades, así como a extranjeros.
Omar Aragón, de la Unica (Nicaragua), explicó que su universidad cuenta con oficina de relaciones internacionales. Las acciones de esta oficina están basadas en el plan estratégico. Atiende convenios, cursos de titulación, jornadas, movilidad, entre otros. Se tienen relaciones con la Universidad Loma Linda (California), en donde ha habido movilidad de estudiantes. Se coordinan servicios e información migratoria para los visitantes extranjeros. Se cuenta con un programa de pasantías pagadas en Disneyword. Invitación a expertos de la internacionalización. Se cuenta con servicios en la web. Se da inducción a visitantes extranjeros sobre cultura nacional. Se cuenta con programas en emprendurismo social e ingeniería humanitaria. "Nos capacitamos mejor para servir mejor" es nuestro lema.

La Ulacit, de Costa Rica, atiende diferentes servicios: Convenios, movilidad de estudiantes y docentes. Contratos con grupos e instituciones proveedoras de servicios educativos, somos inter-mediarios para estudiar en el exterior y en costa Rica.

Mariela Bonilla, del Instituto Tecnológico de Costa Rica (TEC), enfatizó que las oficinas de relaciones internacionales deben ser unidades dedicadas a ofertar servicios a estudiantes, docentes, investigadores, gestores administrativos, etc. Para que exista una cooperación e internacionalización en nuestras universidades, debe existir un presupuesto para ello. Además, se debe contar con personal especializado, así como un sistema y políticas que permitan la movilidad académica y cursos de capacitación. En el TEC de Costa Rica, la oficina tiene una amplia participación: gestión en investigaciones conjuntas, participación en proyectos, pasantías al exterior y profesores invitados, movilidad estudiantil (interna y externa), pasantías internacionales, coordinación de estancias en el exterior, conferencias informativas de orientación, y con organismos, embajadas, universidades extranjeras, divulgación de actividades, ferias de oportunidades estudianti- 
les, reconocimiento de estudios (calificaciones), orientación para cursar estudios en el TEC, trámites migratorios, envío de calificaciones, participación en ferias internacionales, base de datos de familias en donde se puede alojar a los estudiantes, así como apoyo para ubicarse en Costa Rica y los servicios turísticos existentes.

Además, gestiona convenios y apoya las iniciativas de las demás unidades en estas iniciativas, revisando, solicitando información a las contrapartes, envío de documentación, seguimiento de convenios (renovación, informes de convenios, etc.). Así como la búsqueda de programas o proyectos internacionales, y una amplia red de contactos. La oficina de relaciones internacionales, cuenta en el TEC con su página web, así como con servicios de información en línea en inglés y español. Además, brinda apoyo en procesos de acreditación internacional. En cuanto a protocolo, atiende a misiones y visitas oficiales.

Inmaculada Madera, señaló que según la Unesco, la universidad debe ser la conciencia de la sociedad, un lugar donde

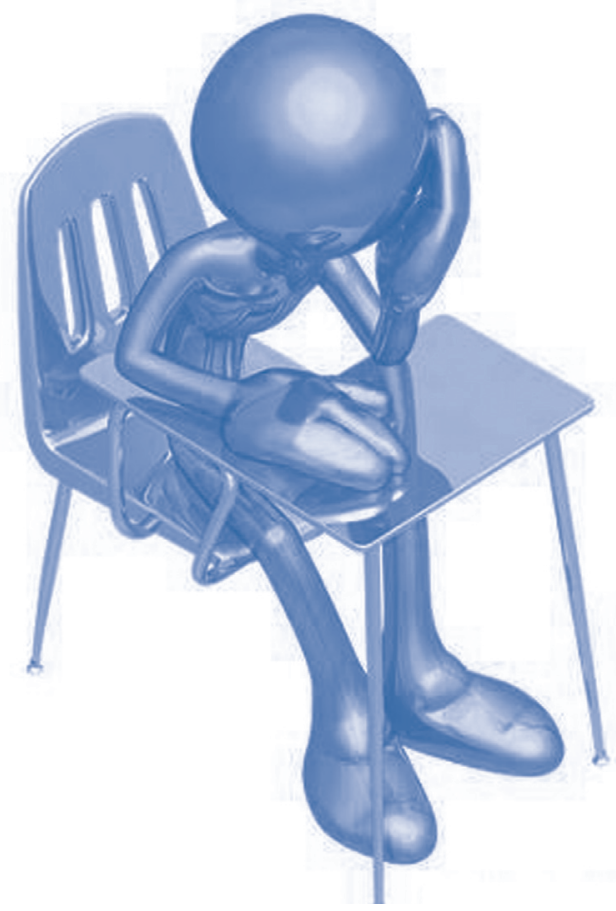

podamos tener una visión más amplia del mundo. Lo anterior es el punto de partida para la internacionalización, la cual debe contar con una visión, una dimensión transformadora como eje transversal, basada en una política con objetivos estratégicos de posicionamiento y gestión estratégica y operativa que conlleve al cumplimiento de objetivos a corto, mediano y largo plazo, así como acciones y presupuesto. Es decir, debe ser un proceso hacia la mejora continua: criterios, indicadores y evaluación, particulares e institucionales. El conjunto de servicios que debe ofrecer una oficina de relaciones internacionales, debe estar orientado a implantar mecanismos y procedimientos que garanticen a la universidad actualización/innovación y calidad/acreditación. Para ello, internamente en la institución, se deben tener propósitos clave como desarrollar la voluntad, la capacidad y la identidad de la comunidad universitaria para la internacionalización. Construir universalidad e identidad planetaria, aprendiendo la interdependencia entre los pueblos. Favorecer la formación de sujetos con las competencias requeridas para accionar con pertinencia ante un entorno cambiante y mundializado. Integrar elementos multidisciplinarios, interdisciplinarios y transdisciplinarios, incorporar a la práctica educativa el uso de las TIC ampliando el impacto global de las instituciones. Lograr mayor pertinencia de calidad en las funciones y la gestión universitarias. Articular los sistemas educativos a partir de criterios más flexibles, que faciliten la compatibilidad. Contribuir al desarrollo local, regional e internacional con visión universal. Se debe estar consciente de que existe un entorno mundial, en donde hay organismos internacionales y regionales donantes, esperando apoyar la educación superior bajo esquemas de integración y políticas de desarrollo.

Justiniano Montenegro explicó que la oficina de relaciones internacionales tiene cuatro años en la Universidad Católica Santa María la Antigua, Panamá. Se cuenta con sesenta convenios efectivos, hay una movilidad por cada año de 
un promedio de veinte estudiantes. Un ejemplo de buenas prácticas en Usma, es el programa internacional en manejo de carga y logística portuaria, que dura cuatro semanas, se desarrolla en siete módulos. La universidad aprovecha la situación geográfica y prácticas económicas del país, además de sus relaciones con otras universidades, gobierno, etc., para su oferta académica internacional y nacional. Se cuenta con las características de un programa internacional. Es un programa integral adaptado que podría iniciarse en cualquier universidad. La oficina de Asuntos Internacionales de la Universidad del Valle de Guatemala" atiende: convenios, investigaciones conjuntas, programas académica compartidos, proyección comunitaria, intercambio, prácticas profesionales. Actualmente se cuentan con 44 convenios y once programas de pasantías internacionales. Hay movilidad académica, se atienden visitantes internacionales, se cuenta con un centro de información. La UVG utiliza las TIC para las diferentes actividades que realiza la ORI. Los intercambios estudiantiles se tienen en los países de: Canadá, España, Estados Unidos y Taiwán.

Los servicios de la ORI son: presentación de programa de intercambio, orientación general en el proceso a estudiantes, profesores y padres de familia, apoyo a recopilación de información y programas académicos de universidad extranjera, orientación y seguimiento para aplicación, gestión administrativa interna para la inscripción como estudiante ausente, convalidación y reinscripción del estudiante. Se atienden visitas internacionales y delegados de instituciones extranjeras, así como profesores o investigadores visitantes, voluntarios en investigación y proyección social, grupos de estudiantes y paquetes especiales.

La ORI coordina con los campus externos para promover y organizar estudios cortos en la UVG. Todos los programas tienen un componente académico, cultural/turístico y uno ecológico. Por ejemplo: programa de desarrollo-enfoque en un proyecto comunitario, programa de idioma español, programa de antropología y sociología y escuela etnográfica. Para centralizar la información sobre las actividades de internacionalización dispersas dentro de la universidad, Ia ORI se establece como un centro de información: recopilación de información, sistematización de información y difusión de información. Existe un amplio desarrollo de base de datos para: redes, visitas internacionales, actividades internacionales, currículos internacionalizados, proyectos de cooperación internacional, publicaciones, servicios a sector privado.

El tema Ects European Credit transfer System fue expuesto por Nizar Ayadi, quien buscó algunas repuestas a este programa. Lo tipificó como un sistema europeo de transferencia (acumulaciones de créditos). Facilita la movilidad, reconocimiento de calificaciones, transparencia entre los sistemas de educación superior, oportunidades de formación de estudiantes en otros países, aprendizaje en toda la vida. No debe ser: definido en función de las horas de docencia, teórica y práctica, impartida por el profesor. Definido en función exclusiva de las horas presenciales. Características: ECTS. Carga por trabajo. 180 ó 240 créditos, durante cuatro años. Carga: tiempo invertido en asistencia a clases, seminario, estudio personal, preparación y realización de exámenes, etc. Se asignan a todos los componentes educativos que incluyen las prácticas. Documentos esenciales de ECTS. Catálogo informativo en dos idiomas, por lo menos, impreso y en la web. Elementos: introducción, información práctica (cómo llegar, costo de vida, etc.), departamento (estructura de los cursos, período, lenguas de enseñanza), glosario y ejemplos. Contrato de estudios: lista de asignaturas, transferencia de créditos. Expediente académico. Documenta todos los resultados del estudiante, notas, etc. La clave es la transparencia, confianza, acuerdo, reconocimiento, flexibilidad, movilidad, diploma supplent, documento anexo a un título de educación superior, proveer descripción de la naturaleza, nivel, contexto, contenido y estatus. 
Lo que vive un estudiante en el extranjero: una nueva casa (una universidad), muchas casas (diferentes universidades). La experiencia se lleva para toda la vida, así lo explicó Aliandra Barlete de la Universidad de Alicante.

¿Cómo desean ustedes que los estudiantes internacionales se recuerden de la experiencia en su universidad? La respuesta a esta pregunta definirá la calidad de los servicios que ustedes van a ofrecer. Obsérvense las diferentes fases por las que atraviesa un estudiante extranjero: Ubicándonos. Diferentes países, clima variado, nacionalidades diferentes y varias mudanzas por correo, que aumentaba el equipaje. Orientación antes de llegar. Visado, dónde vivir, como llega a su casa y a la universidad. Plan de estudios; que va a hacer el estudiante y por cuánto tiempo. Cultural schock, integración, turismo, servicios médicos, dentista, deportes y depresión y saludable. Orientación durante la estadía. Nuevos visados para alargar el tiempo. Soporte institucional, coordinación de programas. Nunca termina la orientación que necesita. Acomodación. Ellos prefieren lugares que lleven las cuentas incluidas (Internet, agua, luz, etc.) Integración.

¿El conjunto de servicios que debe ofrecer una oficina de relaciones internacionales debe estar orientado a implantar mecanismos y procedimientos que garanticen a la universidad actualización/innovación y calidad/acreditación "77
Estudiantes de varios países, pueden realizar encuentros para entretenerse y compartir. Un ejemplo se puede encontrar en: http://www.ua.pt/guiaonlina/Pagelmage.aspx Organización de pequeños grupos con actividades basadas en el interés de los estudiantes: cooking group, table games group, sports group. Bibliotecas, conversatorio, tertulias. International coffe-hour. Nos olvidamos. Rol en la experiencia. La mayoría de estudiantes de grado no han vivido en el extranjero. No han abierto una cuenta bancaria, en su mayoría. Mezcla de sentimientos: aventura, curiosidad y frustración.

Para diseñar los servicios, es necesario hacerse los siguientes interrogantes: ¿Qué tipo de experiencia se desea ofrecer a estudiantes y profesores visitantes? ¿Cuál identidad internacional se desea construir? ¿Cómo se quiere que los estudiantes recuerden a la universidad? Las universidades que envían deberían también orientar a los estudiantes, docentes, investigadores, etc., que viajan.

\section{CONCLUSIONES}

En El Salvador, las dos universidades deberán potenciar los programas de movilidad del docente y estudiantes. Hay que definir estrategias. Por ejemplo, pasantías internacionales. "El ITCR está dispuesto a negociar los términos, para potenciar la movilidad", dijo Maricela Bonilla.

Justiniano Montenegro, de la Usma, Panamá en nombre de todo el equipo agradeció a UES y UTEC por las atenciones. A la institución y a los participantes.

Es una atención a la red. Una oficina de relaciones internacionales deberá considerar los ejemplos para crearla y mejorar los servicios. Los planes estratégicos deben considerar los servicios de relaciones internacionales como un punto importante para la internacionalización. Se deben iniciar las 
acciones y dirigirlas hasta los órganos de poder y de toma de decisiones. Las ideas deberán retomar para nuestros países e instituciones. Se deben aprovechar los espacios que ofrece la red Inca y trabajar en ella.

\section{Fuentes}

- Aragón, Omar. La ORI. Universidad Católica de Managua, Nicaragua. Encuentro Red Inca Julio 2010. San Salvador, El Salvador, América Central.

- Ayadi, Nizar. Internacionalización at home. Promover las relaciones internacionales en su universidad. Selección de socios y negociación de acuerdos. Universidad de Alicante. Encuentro Red Inca 5, 6 y 7 de julio 2010. San Salvador, El Salvador, América Central.

- Barlete, Aliandra. Oficina de Gestión de proyectos internacionales. Universidad de Alicante. Encuentro Red Inca, julio 2010. San Salvador, El Salvador, América Central.

- Bonilla Freer, Maricela. Servicios de internacionalización del Instituto Tecnológico de Costa Rica. Encuentro Red Inca, julio 2010. San Salvador, El Salvador.

- Escarré, Roberto, Director de la Oficina Gestión de Proyectos Internacionales (OGPI) de la Universidad de Alicante. Expuso el tema de Marketing y financiación de la internacionalización en el contexto de la educación, como un tema transnacional. Encuentro Red Inca julio 2010. San Salvador, El Salvador.

Froh, Rebeca. Servicios de internacionalización de la Univer- sidad del Valle de Guatemala. Encuentro Red Inca, julio 2010. San Salvador, El Salvador.

- Hernández, Gaspar. Universidad de Alicante, Oficina de Relaciones Internacionales, "Servicios para la gestión de la movilidad". Encuentro Red Inca, julio 2010. San Salvador, El Salvador.

- Madera, Dra. Luz Inmaculada vicerrectora para Asuntos Internacionales de la Universidad Apec, Santo Domingo, República Dominicana, "Importancia de desarrollar una cultura de internacionalización y cooperación entre el personal de las instituciones". Encuentro Red Inca, julio 2010. San Salvador, El Salvador.

- Mitre, Odilia. Servicio Help Desk. Universidad Tecnológica de Panamá. Encuentro Red Inca Julio 2010. San Salvador, El Salvador.

- Montenegro, Justiniano. Oficina de Relaciones Internacionales. Universidad Católica Santa María la Antigua, Panamá. Encuentro Red Inca, julio 2010. San Salvador, El Salvador.

- Nieto, Ada Ruth, Secretaria de Relaciones Internacionales UES, El Salvador. Encuentro Red Inca, julio 2010. San Salvador, EI Salvador

- Orantes, Blanca Ruth. Directora de Investigaciones Utec, EI Salvador. Encuentro Red Inca, julio 2010. San Salvador, El Salvador.

- www.enic-naric.net europa.eu.int/comm/education/programmes. 